Pacific Journal of Mathematic 


\title{
A FIXED POINT THEOREM FOR MULTI-VALUED FUNCTIONS
}

\author{
L. E. WARD, JR.
}

1. Introduction. If $\mathrm{Y}$ is a topological space then $S(Y)$ denotes the space of closed subsets of $Y$, endowed with the finite topology [2]. We say that a multi-valued function $F: X \rightarrow Y$ is continuous provided $F(x)$ is a closed set for each $x \varepsilon X$, and the induced (single-valued) function $f: X \rightarrow S(Y)$ is continuous in the usual sense. This definition of continuity for multi-valued functions is equivalent to that of Strother [4]. The space $X$ is said to have the F.p.p. (= fixed point property for continuous multi-valued functions) if and only if for each such function $F: X \rightarrow X$, there is an $x \varepsilon X$ such that $x \varepsilon F(x)$. The space $X$ has the f.p.p. if it has the fixed point property for continuous singlevalued functions. It is hardly surprising that the $T_{1}$-spaces with the F.p.p. constitute a fairly small subclass of those with f.p.p. Indeed, Plunkett [3] has shown that a Peano continuum has the F.p.p. if and only if it is a dendrite. It is worth noting that Plunkett's argument employs the convex metric of a dendrite in much the same manner as the author [7] has used the order structure of certain acyclic continua to obtain fixed point theorems. A related argument has been used by Capel and Strother [1] to show that a tree has the fixed point property for continuous multi-valued functions for which the image of each point is connected. Their proof depends on being able to produce a continuous selection, in the sense of Michael, on the class of subcontinua of a tree.

In this paper an order-theoretic characterization of a wide class of acyclic spaces is given. This characterization is in the same spirit as the analogous results of [6] and [7] for trees and generalized trees. It is then shown, using their order properties, that such spaces have the F.p.p. To some extent the argument borrows from all of the proofs cited above.

2. Topologically chained continua. If $X$ is a space and $\leqq$ is a partial order on $X$, we write $L(x)=\{a: a \leqq x\}$ and $M(x)=\{a: x \leqq a\}$. It is natural and convenient to define

$$
[x, y]=M(x) \cap L(y)
$$

and, if $A \subset X$, we write $M(A)$ for the union of all $M(x)$ for which

Received August 7, 1957, and in revised forms November 4, 1957, and February 6, 1958. Presented to the American Mathematical Society, August 29, 1957, 
$x \in A$. An antichain of $X$ is a subset in which no two distinct elements are related under the partial order. A zero of a subset $\mathrm{A}$ of $X$ is a member $a_{0}$ of $A$ such that $A \subset M\left(a_{0}\right)$.

In what follows it will be convenient to use two theorems from [5]. These are stated below as Theorems A and B. The partial-order $\leqq$ is said to be upper (lower) semicontinuous provided $M(x)(L(x))$ is closed, for each $x \in X$. It is semicontinuous if it is both upper and lower semicontinuous.

THEOREM A. If the compact space $X$ is endowed with an upper (lower) semicontinuous partial order then $X$ admits a maximal (minimal) element.

THEOREM B. If the compact space $X$ is endowed with an order-dense semicontinuous partial order and if the set of maximal elements or the set of minimal elements is connected, then $X$ is connected.

Recall that a continuum (= compact connected Hausdorff space) is unicoherent if it cannot be represented as the union of two subcontinua whose intersection is not connected. A continuum is hereditarily unicoherent if each of its subcontinua is unicoherent.

A continuum is topologically chained if each pair of points is contained in a subcontinuum which has exactly two non-cutpoints, and such a subcontinuum is called a topological chain. This concept is a natural generalization of the notion of an arc. Note that, if $x$ and $y$ are distinct elements of a continuum which is topologically chained and hereditarily unicoherent, there is a unique subcontinuum $C(x, y)$ which is irreducible about $x$ and $y$. Moreover, $C(x, y)$ is a topological chain with $\mathrm{x}$ and $y$ for endpoints.

Consider the following five properties enjoyed by some spaces $X$ admitting a partial order, $\leqq$.

I. $[x, y]$ is closed and simply ordered for each $x$ and $y$ in $X$.

II. $\leqq$ is order-dense.

III. There exists $e \in X$ such that $M(e)=X$.

IV. If $x$ and $y$ are points of the subcontinuum $Y$ and $x \leqq y$, then $[x, y] \subset Y$.

V. If $A$ is an antichain of $X$ and $P$ is a continuum contained in $M(A)$, then $P \subset M(x)$ for some $x \in A$.

LeMma 1. If $X$ is a compact Hausdorff space with a partial order satisfying I and II, and if $x<y$ in $X$, then $[x, y]$ is a topological chain, 
Proof. For each $t \in[x, y]$ the sets

$$
\begin{aligned}
L(t) \cap[x, y] & =[x, t], \\
M(t) \cap[x, y] & =[t, y]
\end{aligned}
$$

are closed so that $\leqq$ is semicontinuous on $[x, y]$. Since $[x, y]$ has only one minimal element it is connected by Theorem B. If $x<t<y$ then the continua $[x, t]$ and $[t, y]$ have only the point $t$ in their intersection, so that $x$ and $y$ are the only non-cutpoints of $[x, y]$; that is, $[x, y]$ is a topological chain.

Lemma 2. If $X$ is a compact Hausdorff space with a partial order satisfying I, III, and V, then each continuum contained in $X$ has a zero.

Proof. By I and III the set $L(x)=[e, x]$ is closed for each $x \in X$ and consequently, by Theorem A, each compact subset of $X$ contains a minimal element. If $Y$ is a subcontinuum of $X$ then the set $A$ of minimal elements of $Y$ forms a nonempty antichain. It follows from $V$ that $A$ contains only one element, that is, $Y$ has a zero.

THeOREM 1. A necessary and sufficient condition that $X$ be a topologically chained, hereditarily unicoherent continuum is that $X$ be a compact Hausdorff space which admits a partial order satisfying I-V.

Proof. Necessity. Let $X$ be a topologically chained, hereditarily unicoherent continuum. Fix $e \in X$ and let $x \leqq y$ mean that $x \in C(e, y)$. Then $L(x)=C(e, x)$ and I, II and III are easily verified. If $x \leq y$ and $x$ and $y$ are elements of the subcontinuum $Y$ then, by hereditary unicoherence, $[x, y] \cap Y$ is connected. Since $x$ and $y$ are elements of $Y$ it follows that $[x, y] \subset Y$. This establishes IV. To see that $\mathrm{V}$ is satisfied let $A$ be an antichain of $X$ and suppose that $P$ is a continuum contained in $M(A)$. If $P$ meets $M(x)$ and $M(y)$ where $x$ and $y$ are distinct points of $A$, we may select $p \in M(x) \cap P$ and $q \in M(y) \cap P$. In view of $\mathrm{I}$ the points $p$ and $q$ are not comparable. Therefore, the continua $L(p) \cup P$ and $L(q) \cup P$ meet in the non-connected set $P \cup(L(x) \cap L(y))$, and this contradicts the hereditary unicoherence of $X$.

Sufficiency. Let $X$ be a compact Hausdorff space which admits a partial order satisfying I-V. By Lemma 1 each set $L(x)=[e, x]$ is a topological chain and therefore $X$ is a topologically chained continuum. Now suppose $A$ and $B$ are subcontinua of $X$ and that $x$ and $y$ are distinct elements of $A \cap B$. In order to show that $A \cap B$ is connected and thus that $X$ is hereditarily unicoherent it is sufficient to prove that

$$
Z=[z, x] \cup[z, y] \subset A \cap B,
$$


where $z$ is the supremum of $L(x) \cap L(y)$. The existence of $z$ is assured by Theorem A. By Lemma $2, A$ has a zero, $a_{0}$, so that $a_{0}$ is a predecessor of $x$ and $y$ and hence also of $z$. By IV,

$$
[z, x] \subset\left[a_{0}, x\right] \subset A \text {. }
$$

It is clear that, by a sequence of analogous arguments, $Z \subset A \cap B$.

3. The fixed point theorem. The proof of Theorem 2 which follows is patterned after that of Theorem 10 in [7] where it was assumed that the partial order had a closed graph. In view of our weaker hypotheses it has been necessary to revise that argument extensively. For the remainder of this paper the term sequence is used in its generalized sense, that is, a function defined on the predecessors of some ordinal number. If $x$ is a sequence then a subsequence of $x$ is the restriction of $x$ to some cofinal subset of its domain.

A useful continuity property of a partial order satisfying I-V is given by the following lemma.

Lemma 3. Let $X$ be a compact Hausdorff space which contains no indecomposable continuum and which admits a partial order satisfying $\mathrm{I}-\mathrm{V}$. If $x$ is an increasing sequence in $X$ then $\lim x$ exists and $x_{a} \leqq \lim$ $x$ for each $\alpha$ in the domain of $x$.

Proof. Let $x_{0}$ be a limit point of $x$ and suppose $x_{0} \in X-M\left(x_{\beta}\right)$ for some $\beta$. Without loss of generality we may take $\beta=1$. Let $C$ be the union of the topological chains $\left[x_{\alpha}, x_{\alpha^{\prime}}\right], \alpha^{\prime}>\alpha$. If $\sigma$ is the zero of $\bar{C}$ then $x_{0} \in \bar{C}$ implies $\sigma<x_{1}$. If $K$ is a subcontinuum of $\bar{C}$ which contains the values of some subsequence of $x$, then by IV, $x_{\alpha} \in K$ implies $\left[x_{\alpha}, x_{\alpha}{ }^{\prime}\right] \subset K$ for each $\alpha^{\prime}>\alpha$ and hence $K$ contains $x_{0}$ and $\sigma$. Again by IV, $K=\bar{C}$. It follows that $\bar{C}$ is the union of no two proper subcontinua and this contradicts our hypothesis that $X$ contains no indecomposable continuum. Hence $x_{\alpha} \leqq x_{0}$ for each $\alpha$ in the domain of $x$. If $y \in X$ is not a predecessor of $x_{0}$ then $y$ is a member of the open set $U=X-L\left(x_{0}\right)$. Since $x_{\alpha} \in X-U$ for each $\alpha, y$ is not a limit point of $x$. Therefore $x_{0}$ is the unique limit point of $x$, that is, $x_{0}=\lim x$.

Lemma 4. Let $K$ be a connected topological space and let $F$ be a continuous multi-valued function defined on $K$. Suppose that $F(x)$ is a compact set for each $x \in K$. If $Q$ is a quasi-component of $F(K)$ then $F(x) \cap Q$ is nonempty for each $x \in K$.

Proof. By definition $Q=\cap\left\{V_{\alpha}\right\}$ where $\left\{V_{\alpha}\right\}$ is the family of open and closed sets containing $Q$. If $F(x)$ meets each $V_{\alpha}$ then, by the 
compactness of $F(x)$, it meets $Q$ and therefore $F^{-1}(Q)=\cap\left\{F^{-1}\left(V_{\alpha}\right)\right\}$. By an elementary continuity argument it can be verified that each set $F^{-1}\left(V_{\alpha}\right)$ is both open and closed and hence is equal to $K$. Therefore, $K=F^{-1}(Q)$ and the lemma follows.

For the remainder of this section, $X$ denotes a topologically chained, hereditarily unicoherent continuum which contains no indecomposable subcontinuum, and $F: X \rightarrow X$ is a continuous multi-valued function. By Theorem 1 the space $X$ admits a partial order satisfying I-V. Let $J$ be the set of all elements $x$ of $X$ such that (i) there exists a minimal element $t_{x}$ in the set $F(x) \cap M(x)$, and (ii) if $x<p \leqq t_{x}$ then $F(p)$ and $\left[p, t_{x}\right]$ are disjoint.

Lemma 5. If $F$ is fixed point free then $J$ is not empty. Indeed if $a \in X$ such that $F(a) \cap M(a)$ is not empty then $M(a) \cap J$ is not empty.

Proof. By Lemma 2, $X$ has a zero and hence there exists $a \in X$ such that $F(a) \cap M(a)$ is not empty. If $b \in F(a) \cap M(a)$ then by Theorem A there is a minimal element $t_{a}$ in the compact set $F(a) \cap[a, b]$. Clearly $t_{a}$ is minimal in $F(a) \cap M(a)$. Let $K$ denote the set of all $p \in\left[a, t_{a}\right]$ such that $F(p) \cap\left[p, t_{a}\right]$ is not empty. Then $a \in K$ and $t_{a} \in X-K$. If $x_{0}=\sup K$ it is easy to construct an increasing sequence $y$ in $K$ such that $\lim y=x_{0}$. For each $\alpha$ in the domain of $y$ let $z_{\alpha} \in F\left(y_{\alpha}\right) \cap\left[y_{\alpha}, t_{a}\right]$. Then the sequence $z$ has a limit point $z_{0}$ and by continuity $z_{0} \in F\left(x_{0}\right)$. Further, since $y_{\alpha}<z_{\alpha} \leqq t_{a}$ for each $\alpha$, it follows that $z_{0} \in\left[x_{0}, t_{a}\right]$ and hence $x_{0} \in K$. Now let $t_{0}$ be minimal in $F\left(x_{0}\right) \cap\left[x_{0}, z_{0}\right]$. If $x_{0}<p \leqq t_{0}$ and $F(p) \cap\left[p, t_{0}\right]$ is not empty then $t_{0} \leqq t_{a}$ implies that $F(p) \cap\left[p, t_{a}\right]$ is not empty. But this is a contradiction since $x_{0}$ is the supremum of $K$. Therefore $F(p)$ and $\left[p, t_{0}\right]$ are disjoint, that is, $x_{0} \in J$ and the lemma is proved.

Consider the set $S$ of all sequences $x$ such that $J$ contains the range of $x$ and

$$
\begin{gathered}
x_{1}<x_{2}<\cdots<x_{\alpha}<\cdots, \\
t_{\alpha} \leqq x_{\alpha+1} \text { for each } \alpha+1 \text { in the domain of } x,
\end{gathered}
$$

where $t_{\alpha}=t_{x_{\alpha}}$. If $J$ is not empty then $S$ contains at least one nonempty sequence. We partially order the elements of $S$ in the following manner : $x$ precedes $y$ provided $x$ is an initial segment of $y$. Clearly, if $N$ is a simply ordered subset of $S$ then $U N$ is a member of $S$. By Zorn's lemma $S$ contains a maximal element.

Lemma 6. If $F$ is fixed point free and $x$ is an element of $S$ then the domain of $x$ is not a limit ordinal. 
Proof. If the domain of $x$ is a limit ordinal, let $x_{0}=\lim x$, $t_{0}=\lim t$. By Lemma $3, x_{\alpha} \leqq x_{0}$ and $t_{\alpha} \leqq t_{0}$ for each $\alpha$. From the definition of $J$ we have $x_{\alpha}<t_{\alpha}$ for each $\alpha$ and hence $x_{0} \leqq t_{0}$. But by the definition of $S, t_{\alpha+1} \leqq x_{\alpha}$ for each $\alpha$ and hence $t_{0} \leqq x_{0}$. This implies that $x_{0}=t_{0}$ is a fixed point of $F$, which is a contradiction.

LEMMA 7. If $F$ is fixed point free and $x$ is an element of $S$ then the domain of $x$ is finite.

Proof. Suppose the domain of $x$ is not finite. We denote by $\omega$ the first infinite ordinal. Then by Lemma 6, $\omega$ is a member of the domain of $x$. From the definition of $S$ it is clear that $x$, restricted to $\omega$, is an element of $S$, contrary to Lemma 6 .

THEOREM 2. Each topologically chained, hereditarily unicoherent continuum which contains no indecomposable continuum has the F.p.p.

Proof. If $F: X \rightarrow X$ is fixed point free then, by Lemma $5, J$ is not empty and hence $S$ contains a maximal element, $x$. By Lemma 7 the domain of $x$ is a set $\{1,2, \cdots, N\}$ of integers. We write $t_{N}=t_{x_{N}}$. By Lemma 5 and the maximality of $x$ it follows that $M\left(t_{N}\right) \cap F\left(t_{N}\right)$ is empty. Now from Corollary 9.6 of [2] the set $F\left(\left[x_{N}, t_{N}\right]\right)$ is compact and hence its components and quasi-components are identical. Let $D$ be that component of $F\left(\left[x_{N}, t_{N}\right]\right)$ such that $t_{N} \in D$. By Lemma 4, $F\left(t_{N}\right) \cap D$ is not empty and hence $D-M\left(t_{N}\right)$ is not empty. Now $D$ is a continuum and hence has a zero $z$ which precedes $t_{N}$. By IV, $\left[z, t_{N}\right] \subset D$ but since $F$ is fixed point free there exists $q \in X$ with $x_{N}<q<t_{N}$ such that $\left[q, t_{N}\right] \subset D-F\left(t_{N}\right)$. Let $b$ be an infinite increasing sequence in $\left[q, t_{N}\right]$ such that $\lim b=t_{N}$. For each $b_{a}$ there exists $a_{a}, x_{N}<a_{x}<t_{N}$, such that $b_{\alpha} \in F\left(a_{\alpha}\right)$. Since $x_{N} \in J$ we infer that $b_{\alpha}<a_{\alpha}$. Clearly lim $a=t_{N}$ from which it follows that $t_{N} \in F\left(t_{N}\right)$, a contradiction. Therefore $F$ cannot be fixed point free.

4. Remarks. The following question is of some interest. Can the assumption of hereditary decomposability be omitted from the hypotheses of Theorem 2? This assumption is essential to Lemma 3 so that an affirmative answer to our question would require a substantially different proof. Even more difficult is the problem of characterizing the F.p.p. among the topologically chained continua. In view of Plunkett's characterization [3] of the F.p.p. for Peano continua, there would seem to be some hope of discovering a succinct characterization. 


\section{REFERENCES}

1. C. E. Capel and W. L. Strother, $A$ new proof of a fixed point theorem of Wallace, Bull. Amer. Math. Soc. Abstract 62-1-111.

2. E. Michael, Topologies on spaces of subsets, Trans. Amer. Math. Soc., 71 (1951), 152182.

3. R. L. Plunkett, A fixed point theorem for continuous multi-valued transformations, Proc. Amer. Math. Soc., 7 (1956) 160-163.

4. W. L. Strother, On an open question concerning fixed points, Proc. Amer. Math. Soc., 4 (1953), 988-993.

5. L. E. Ward, Jr., Partially ordered topological spaces, Proc. Amer. Math. Soc., 5 (1954), 144-161.

6. $\quad$, A note on dendrites and trees, Proc. Amer. Math. Soc., 5 (1954), 992-994.

7. _ - Mobs, trees, and fixed points, Proc. Amer. Math. Soc., 8 (1957), 798-804.

U. S. Naval Ordnance Test Station

ChINA LAKE, CALIFORNIA 



\section{PACIFIC JOURNAL OF MATHEMATICS}

\section{EDITORS}

\section{David Gilbarg}

Stanford University

Stanford, California

\section{R. A. Beaumont}

University of Washington

Seattle 5, Washington

\author{
A. L. Whiteman
}

University of Southern California Los Angeles 7, California

E. G. Straus

University of California

Los Angeles 24, California

\section{ASSOCIATE EDITORS}

\author{
E. F. BECKENBACH \\ C. E. BURGESS \\ M. HALL \\ E. HEWITT
}
A. HORN
V. GANAPATHY IYER
R. D. JAMES
M. S. KNEBELMAN
L. NACHBIN
I. NIVEN
T. G. OSTROM
H. L. ROYDEN

M. M. SCHIFFER

G. SZEKERES

F. WOLF

K. YOSIDA

\section{SUPPORTING INSTITUTIONS}

\author{
UNIVERSITY OF BRITISH COLUMBIA \\ CALIFORNIA INSTITUTE OF TECHNOLOGY \\ UNIVERSITY OF CALIFORNIA \\ MONTANA STATE UNIVERSITY \\ UNIVERSITY OF NEVADA \\ OREGON STATE COLLEGE \\ UNIVERSITY OF OREGON \\ OSAKA UNIVERSITY \\ UNIVERSITY OF SOUTHERN CALIFORNIA
}

\author{
STANFORD UNIVERSITY \\ UNIVERSITY OF TOKYO \\ UNIVERSITY OF UTAH \\ WASHINGTON STATE COLLEGE \\ UNIVERSITY OF WASHINGTON \\ * * * * \\ AMERICAN MATHEMATICAL SOCIETY \\ CALIFORNIA RESEARCH CORPORATION \\ HUGHES AIRCRAFT COMPANY \\ THE RAMO-WOOLDRIDGE CORPORATION
}

Mathematical papers intended for publication in the Pacific Journal of Mathematics should be typewritten (double spaced), and the author should keep a complete copy. Manuscripts may be sent to any one of the four editors. All other communications to the editors should be addressed to the managing editor, E. G. Straus at the University of California, Los Angeles 24, California.

50 reprints per author of each article are furnished free of charge; additional copies may be obtained at cost in multiples of 50 .

The Pacific Journal of Mathematics is published quarterly, in March, June, September, and December. The price per volume (4 numbers) is $\$ 12.00$; single issues, $\$ 3.50$. Back numbers are available. Special price to individual faculty members of supporting institutions and to individual members of the American Mathematical Society: $\$ 4.00$ per volume; single issues, $\$ 1.25$.

Subscriptions, orders for back numbers, and changes of address should be sent to Pacific Journal of Mathematics, 2120 Oxford Street, Berkeley 4, California.

Printed at Kokusai Bunken Insatsusha (International Academic Printing Co., Ltd.), No. 6, 2-chome, Fujimi-cho, Chiyoda-ku, Tokyo, Japan.

PUBLISHED BY PACIFIC JOURNAL OF MATHEMATICS, A NON-PROFIT CORPORATION

The Supporting Institutions listed above contribute to the cost of publication of this Journal, but they are not owners or publishers and have no responsibility for its content or policies. 


\section{Pacific Journal of Mathematics}

\section{Vol. 8, No. $4 \quad$ June, 1958}

Richard Arens, The maximal ideals of certain functions algebras ........ 641

Glen Earl Baxter, An operator identity ........................... 649

Robert James Blattner, Automorphic group representations ........... 665

Steve Jerome Bryant, Isomorphism order for Abelian groups ............ 679

Charles W. Curtis, Modules whose annihilators are direct summands...... 685

Wilbur Eugene Deskins, On the radical of a group algebra ............ 693

Jacob Feldman, Equivalence and perpendicularity of Gaussian

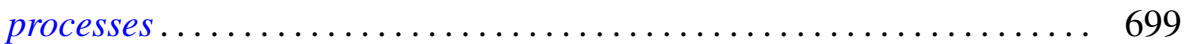

Marion K. Fort, Jr. and G. A. Hedlund, Minimal coverings of pairs by triples....................................... 709

I. S. Gál, On the theory of $(m, n)$-compact topological spaces ......... 721

David Gale and Oliver Gross, A note on polynomial and separable games........................................ 735

Frank Harary, On the number of bi-colored graphs ............... 743

Bruno Harris, Centralizers in Jordan algebras ................... 757

Martin Jurchescu, Modulus of a boundary component ............... 791

Hewitt Kenyon and A. P. Morse, Runs . . . . . . . . . . . . . . . . . . . . . . 811

Burnett C. Meyer and H. D. Sprinkle, Two nonseparable complete metric

spaces defined on $[0,1] \ldots \ldots \ldots \ldots \ldots \ldots \ldots \ldots \ldots \ldots \ldots . \ldots . \ldots . \ldots . \ldots 25$

M. S. Robertson, Cesàro partial sums of harmonic series expansions...... 829

John L. Selfridge and Ernst Gabor Straus, On the determination of numbers by their sums of a fixed order ........................ 847

Annette Sinclair, A general solution for a class of approximation

problems .................................

George Szekeres and Amnon Jakimovski, $(C, \infty)$ and $(H, \infty)$ methods of summation...................................... 867

Hale Trotter, Approximation of semi-groups of operators. ............. 887

L. E. Ward, A fixed point theorem for multi-valued functions ........... 921

Roy Edwin Wild, On the number of lattice points in $x^{t}+y^{t}=n^{t / 2} \ldots \ldots .929$ 